\title{
Modelling ultrasound waves bubble formation in ethanol/ethyl acetate azeotrope mixture
}

\author{
Olagoke Oladokun ${ }^{1,2}$, Arshad Ahmad, ${ }^{1, *}$, Adnan Ripin ${ }^{1}$, Tuan A. T. Abdullah', Bemgba B. \\ Nyakuma $^{1}$, Nur Amira Hadi ${ }^{1}$, Ali H. Al-Shatri ${ }^{1}$, Murtala Ahmed ${ }^{2}$, Habib Alkali ${ }^{1,2}$, and Aliyu \\ A. Bello ${ }^{1,2}$ \\ ${ }^{1}$ Centre of Hydrogen Energy, Institute of Future Energy, Universiti Teknologi Malaysia, 81310 Johor \\ Bahru, Johor, Malaysia. \\ ${ }^{2}$ Department of Chemical Engineering, University of Maiduguri, Nigeria.
}

\begin{abstract}
The separation of an azeotropic mixture such as ethanol/ethyl acetate in distillation process can be enhanced by ultrasound wave. The application of ultrasound wave creates bubble cavitation in the mixture and shifts the vapour-liquid equilibrium favouring the separation of the azeotropic mixture. This study investigates the formation of bubbles in the mixture through modelling and simulation. The results obtained show that bubble formation at low ultrasound frequency is favoured by the increase in intensity, which has a direct relation to sonic pressure. The optimal sonic pressure for bubble formation at equilibrium is $5 \mathrm{~atm}$ and conforms to the model for small bubble formation with radius of $0.14 \mu \mathrm{m}$. Furthermore, the maximum possible number of bubbles at equilibrium in the ethanol/ethyl acetate azeotropic mixture of $1 \mathrm{~L}$ is $91 \times 10^{\text {s. }}$. The developed model can be used to determine the optimal sonic pressure, sound intensity, size of bubble, and possible number of bubbles formed at equilibrium.
\end{abstract}

\section{Introduction}

The formation of bubbles in liquid when subjected to ultrasound wave is an important application in the field of sonochemistry. The practical application of sonochemistry process includes and not limited to chemical reaction [1], ultrasonic cleaning [2], and process intensification [3, 4], such as separation of an azeotropic mixture [5, 6]. Mahdi et al. [7] worked on the intensification of distillation process for the separation of ethanol/ethyl acetate azeotropic mixture using ultrasound wave. Other researchers have developed valid mathematical theories behind acoustic cavitation [5, 8, 9]. However, the formation of bubbles in relation to the quantity of bubbles formed during acoustic cavitation is missing in the literature. Consequently, this study investigates modelling and simulation of bubble formation, bubble type, and the number of bubbles in an azeotropic mixture of ethanol/ethyl acetate. The separation of azeotropic mixtures using ultrasound wave can be optimised by understanding the science of bubble formation in relation to the type of bubble and the number of bubbles expected and subsequently, the rise and collapse of the bubbles. 


\section{Method}

The ethanol/ethyl acetate azeotropic mixture was exposed to ultrasound wave from a transducer as shown in Figure 1. Bubbles start to form in the mixture at certain sound intensity and frequency [10].

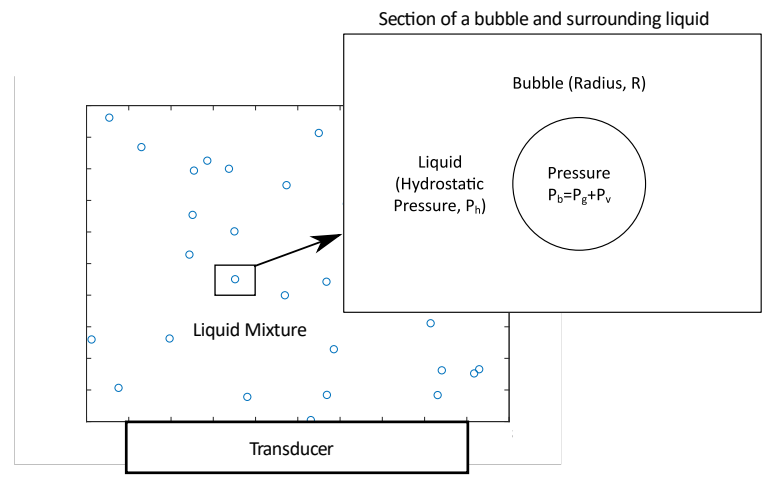

Fig. 1. Ultrasound-enhanced bubble formation in a liquid mixture.

\subsection{Bubble formation theory}

The relationship between bubble radius and ultrasound wave pressure at equilibrium is essential in determining the feasible number of bubbles formed. The theory behind the relationship is established as follows [1, 10-12].

A bubble at equilibrium in a liquid is neither contracting (external forces) nor expanding (internal forces), where the radius of the bubble is denoted as $R_{E}$. The external forces (liquid pressure, $P_{\iota}$ ) are balanced by the internal forces (bubble pressure, $P_{b}$ ) (refer to Figure 1).

The liquid pressure contracting the bubble external wall is the combination of the liquid hydrostatic pressure $\left(P_{n}\right)$ and the surface tension effect $\left(2 \sigma / R_{E}\right)$, as shown in Equation 1 :

$$
P_{L}=P_{h}+2 \sigma / R_{o}
$$

Where the bubble pressure is expressed as the sum of pressures from trapped gas $\left(P_{s}\right)$ and vapour in the bubble $\left(P_{v}\right)$ (Equation (2)):

$$
P_{b}=P_{g}+P_{v}
$$

At equilibrium and using the subscript $\mathrm{E}$ to denote equilibrium:

$$
\begin{gathered}
P_{L}=P_{b} \\
P_{h}+2 \sigma /_{R_{E}}=P_{g, E}+P_{v, E}
\end{gathered}
$$

Assuming the bubble changes to radius R due to changes in the hydrostatic pressure $\left(P_{h}\right)$, the new gas pressure $P_{s}[1]$ can be defined as:

$$
P_{g}=P_{g, E}\left(R_{E} / R\right)^{3 \gamma}
$$

Where $\gamma$ is the ratio of heat capacity at constant pressure $\left(C_{p}\right)$ and volume $\left(C_{\text {v }}\right)$.

Rewriting Equation 4 to account for the new change gives Equation 6: 


$$
P_{h}+2 \sigma /_{R}=P_{g, E}\left(R_{E} / R\right)^{3 \gamma}+P_{v, E}
$$

A critical hydrostatic pressure where any changes lead to rapid change in bubble radius is given by Equations 7-9 [1, 10]:

$$
d P_{h} / d R=0
$$

The critical radius $R_{\kappa}$ is given by Equation 8 [10]:

$$
R_{K}^{2}=3 / 2 \sigma P_{g, E} R_{E}^{3}
$$

The corresponding critical pressure is presented in Equation 9:

$$
P_{K}=P_{g, E}\left(R_{E} / R_{K}\right)^{3 \gamma}+P_{v, E}-2 \sigma / R_{K}
$$

Substituting for $R_{k}$ from Equation 8 and $\gamma=1$ for an isothermal system:

$$
P_{K}=P_{v, E}-\frac{2}{3}\left\{\frac{\left(2 \sigma / R_{E}\right)^{3}}{3 P_{g, E}}\right\}^{0.5}
$$

Substituting for $P_{s E}$ from Equation 4:

$$
P_{K}=P_{v, E}-\frac{2}{3}\left\{\frac{\left(2 \sigma / R_{E}\right)^{3}}{3\left(P_{h}+2 \sigma / R_{E}-P_{v, E}\right)}\right\}^{0.5}
$$

If the vapour pressure is negligible, $P_{v e} \sim 0$. Equation 11 is written as Equation 12:

$$
P_{K}=-\frac{2}{3}\left\{\frac{\left(2 \sigma / R_{E}\right)^{3}}{3\left(P_{h}+2 \sigma / R_{E}\right)}\right\}^{0.5}
$$

Equation 12 shows that a negative pressure (rarefaction) is needed in the liquid to produce a bubble of radius $R_{t}$. The critical pressure is the sum of hydrostatic pressure $\left(P_{n}\right)$ and Blake threshold pressure $\left(P_{n}\right)[1]$ :

$$
P_{K}=P_{h}+\left(-P_{B}\right)
$$

Further analysis on determining the Blake threshold pressure, which is excess pressure needed above the hydrostatic pressure for the formation of either large or small bubbles, is expressed in Equations 14-16:

$$
P_{B}=P_{h}+\frac{2}{3}\left\{\frac{\left(2 \sigma / R_{E}\right)^{3}}{3\left(P_{h}+2 \sigma / R_{E}\right)}\right\}^{0.5}
$$

For large bubbles $\left(2 \sigma / R_{\mathrm{E}} \ll P_{h}\right)$ :

$$
P_{B} \sim P_{h}+\frac{8 \sigma}{9}\left\{\frac{3 \sigma}{2 P_{h} R_{E}^{3}}\right\}^{0.5}
$$

For small bubbles $\left(2 \sigma / R_{\mathrm{E}} \gg P_{h}\right)$ :

$$
P_{B} \sim P_{h}+0.77 / R_{E}
$$


Furthermore, the relationships between acoustic pressure $\left(P_{A}\right)$, acoustic intensity, $(I)$ acoustic frequency $(f)$, bubble radius $(R)$, and maximum radius $\left(R_{\max }\right)$ of a bubble in the liquid are expressed in Equations 17-19 [1, 10, 13]:

$$
\begin{gathered}
I=\frac{P_{A}{ }^{2}}{2 \rho c} \\
f=\frac{1}{2 \pi R}\left[\frac{3 \gamma P_{h}}{\rho}\right]^{1 / 2}
\end{gathered}
$$

Where $\mathrm{Q}$ is the density of liquid and $c$ is the speed of sound in a liquid:

$$
R_{\text {max }}=\frac{4}{6 \pi f}\left(P_{A}-P_{h}\right)\left(\frac{2}{\rho P_{A}}\right)^{1 / 2}\left[1+\frac{2\left(P_{A}-P_{h}\right)}{3 P_{h}}\right]^{1 / 3}
$$

\subsection{Bubble formation in ethanol/ethyl acetate azeotrope}

Table 1 lists various parameters used in the simulation, essentially the physical properties of the ethanol/ethyl acetate azeotropic mixture and the properties of ultrasound wave. The simulation of the mathematical model equations was carried out in MATLAB 2018.

Table 1. Properties of ethanol, ethyl acetate, and ultrasound wave.

\begin{tabular}{|l|r|r|r|}
\hline & $\begin{array}{c}\text { Ethanol } \\
(\text { EtOH) }\end{array}$ & $\begin{array}{c}\text { Ethyl } \\
\text { Acetate } \\
(\text { EtAc })\end{array}$ & ETOH/ETAC \\
\hline Boiling Point $\left({ }^{\circ} \mathbf{C}\right)$ & 78.4 & 77.11 & 72.8 \\
\hline Density @ 20 ${ }^{\circ} \mathbf{C}\left(\mathbf{g ~ c m}^{-3}\right)$ & 0.789 & 0.9006 & 0.84 \\
\hline Surface Tension at $\mathbf{~ 2 0}^{\circ} \mathbf{C}\left(\right.$ dyn $\left._{\mathbf{~ c m}}\right)$ & 22.39 & 23.75 & 23 \\
\hline Azeotrope Point & 0.56 & 0.54 & 0.54 \\
\hline Relative Volatility & & & 2.28 \\
\hline & & & 1185.4 \\
\hline Ultrasonic Velocity $\left(\boldsymbol{m} \mathbf{s}^{-t}\right)$ & 1180 & & 25 \\
\hline Ultrasound Frequency $(\mathbf{k H z})$ & & & $1-10$ \\
\hline Acoustic Pressure $(\boldsymbol{a t m})$ & & & 1 \\
\hline Mixture Volume $(\boldsymbol{L})$ & & &
\end{tabular}

In other to determine the number of bubbles formed, several assumptions were made and a mathematical function was developed to compute the ideal number of bubbles formed in a volume of liquid.

The number of bubbles $(n)$ is a function of the concentration of air $\left(n_{A i r}\right)$ in the liquid, the volume of liquid $\left(V_{l}\right)$, the volume of the vessel $\left(V_{V}\right)$, and the volume of bubble $\left(V_{b}\right)$.

$$
\begin{gathered}
n=f\left(n_{\text {Air }}, V_{l}, V_{V}, V_{b}\right) \\
V_{b}=4 / 3 \pi R^{3}
\end{gathered}
$$

The assumptions of the simulation are as follows:
i. $\quad 100 \%$ air concentration in the liquid $\left(n_{\text {Air }}\right)$.
ii. Bubble collapse and agglomeration are not considered.
iii. Condition favours self-sustaining bubbles. 
iv. Bubbles are of spherical shape.

v. The volume of liquid equals the sum of bubble volume $\left(V_{l}=\sum V_{b}\right)$. The volume of bubbles is constrained by the volume of liquid available to form the bubble surface boundary.

The simulation algorithm uses the following equations:

i. Equations 14, 15, and 16 are used to determine the type of bubble.

ii. Equation 19 is used to determine the maximum radius of bubble for a given ultrasound intensity.

iii. Equations 20 and 21 are used to determine the number of bubbles in a volume of a liquid.

\section{Results and discussion}

\subsection{Bubble type}

The results of the simulation are presented in this section. Figure 2 shows the profile of Blake threshold pressure (acoustic pressure) and the radius of bubbles formed at equilibrium for general $\left(R_{E}\right)$, large $\left(R_{E}(L B)\right)$, and small $\left(R_{E}(S B)\right)$ bubble types.

The simulation result shows that the general $\left(R_{E}\right)$ bubble profile at equilibrium is closely similar to the small bubble profile, $R_{E}(S B)$. Therefore, the type of bubbles expected to be formed in an ethanol/ethyl acetate azeotropic mixture in ultrasonic medium of $25 \mathrm{kHz}$ is small bubbles.

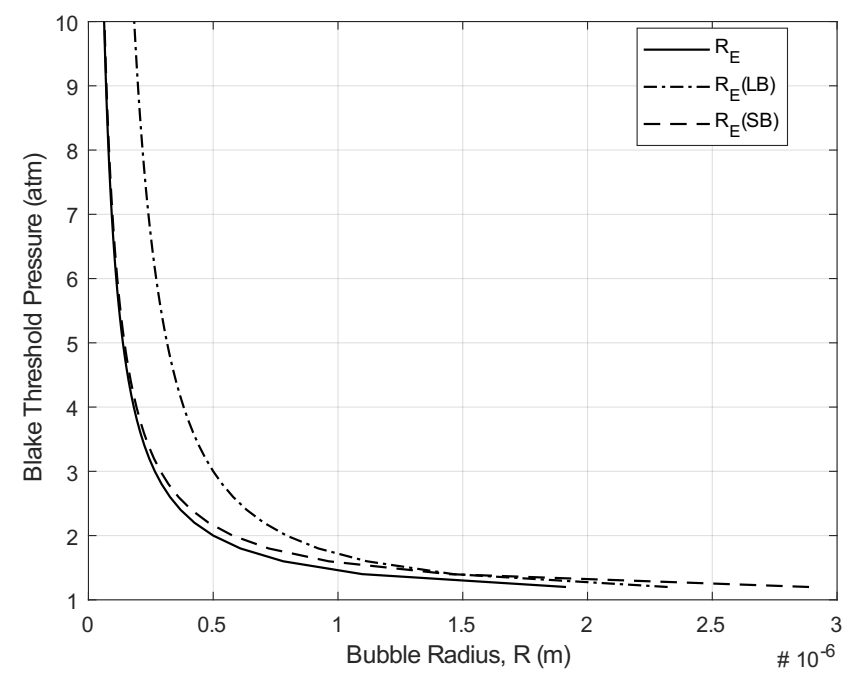

Fig. 2. Effect of ultrasound wave pressure on bubble radius.

\subsection{Number of bubbles}

The results for the ideal number of bubbles formed based on the assumptions highlighted in Section 2.2 and varying acoustic pressure are tabulated in Table 2. 
Table 2. Number of bubbles formed at equilibrium with acoustic pressure of $25 \mathrm{kHz}$.

\begin{tabular}{|r|r|c|r|c|r|r|r|}
\hline \multicolumn{1}{|c|}{$\begin{array}{c}\mathbf{P} \\
(\mathrm{atm})\end{array}$} & \multicolumn{1}{|c|}{$\begin{array}{c}\mathbf{R}_{\mathrm{F}} \\
\left(\mathrm{kW} \mathrm{m}^{2}\right)\end{array}$} & $\begin{array}{c}\mathbf{R}_{\mathrm{E}}(\mathbf{S B}) \\
(\mu \mathrm{m})\end{array}$ & $\begin{array}{c}\mathbf{R}_{\max } \\
(\mu \mathrm{m})\end{array}$ & $\begin{array}{c}\mathbf{n} \\
\left(\times 10^{15}\right)\end{array}$ & $\begin{array}{c}\mathbf{n}(\mathbf{S B}) \\
\left(\times 10^{15}\right)\end{array}$ & $\begin{array}{c}\mathbf{n}_{\max } \\
\left(\times 10^{6}\right)\end{array}$ \\
\hline 2 & 21 & 0.50 & 0.58 & 110.53 & 2 & 1 & 177 \\
\hline 4 & 82 & 0.18 & 0.19 & 285.22 & 40 & 33 & 10 \\
\hline $\mathbf{5}$ & $\mathbf{1 2 9}$ & $\mathbf{0 . 1 4}$ & $\mathbf{0 . 1 4}$ & $\mathbf{3 6 3 . 6 8}$ & $\mathbf{9 1}$ & $\mathbf{8 9}$ & $\mathbf{5}$ \\
\hline 6 & 186 & 0.11 & 0.12 & 438.75 & 173 & 155 & 3 \\
\hline 7 & 253 & 0.09 & 0.10 & 511.26 & 293 & 268 & 2 \\
\hline 8 & 330 & 0.08 & 0.08 & 581.72 & 460 & 425 & 1 \\
\hline 10 & 515 & 0.06 & 0.06 & 717.78 & 962 & 903 & 1 \\
\hline
\end{tabular}

Considering the equilibrium radius $\left(R_{E}\right)$, the result shows that change in acoustic pressure, which is proportional to ultrasound intensity, generates inverse change in bubble equilibrium radius. Consequently, the number of bubbles in a given liquid volume increases exponentially. However, an increase in the acoustic pressure shows corresponding increase in the maximum radius of bubble $\left(R_{m x}\right)$ and decrease in the number of bubbles.

The acoustic pressure of $5 \mathrm{~atm}$ and acoustic intensity of $129 \mathrm{~kW} \mathrm{~m}$ are the optimum conditions for a stable bubble at equilibrium, in which the general and small bubble types are approximately equal $(0.14 \mu \mathrm{m})$ and $2 \sigma / R_{\mathrm{E}} \gg P_{h}$ (refer to Figure 2). At this optimum operating conditions, 91 quadrillion ideal number of bubbles are formed in EtOH/EtAc azeotrope of $1 \mathrm{~L}$. Furthermore, at the frequency of $25 \mathrm{kHz}$ and acoustic pressure of $5 \mathrm{~atm}$, the maximum radius of any bubble formed is $363.68 \mu \mathrm{m}$ and the maximum ideal number of bubble is 5 .

\section{Conclusion}

The model of bubble formation in a liquid when exposed to ultrasound wave was successfully developed. The model was further used to determine the number of bubbles formed in the liquid. The simulation shows that ethanol/ethyl acetate azeotropic mixture conforms to small bubble model. The developed model has the capacity to assist in understanding the science of bubble formation, size, and count in relation to ultrasound wave intensity, frequency, and liquid mixture, consequently leading to sonochemistry process optimisation.

The authors wish to acknowledge the financial and material support from Universiti Teknologi Malaysia and the Ministry of Higher Education (MoHE) Malaysia through the MTAJA grant (Vot. No. 4C116).

\section{References}

1 Mason, T.J., J.P. Lorimer, Applied Sonochemistry: Uses of Power Ultrasound in Chemistry and Processing (2002)

2 Juschke, M., C. Koch. Ultrason. Sonochem. 19, 787 (2012).

3 Kiss, A.A., R. Geertman, M. Wierschem, et al. J. Chem. Technol. Biotechnol. 93, 1219 (2018).

4 Mahdi, T., A. Ahmad, A. Ripin, M.M. Nasef. Chem. Prod. Process Model. 10, (2015). 
5 Zhang, Y., Z. Guo, Y. Gao, X. Du. Ultrasonics sonochemistry. 40, 40 (2018).

6 Mahdi, T., A. Ahmad, M.M. Nasef, A. Ripin. Separation \& Purification Reviews. 44, 308 (2014).

7 Mahdi, T., A. Ahmad, A. Ripin, T.A. Abdullah, M.M. Nasef, M.W. Ali. Ultrasonics sonochemistry. 24, 184 (2015).

8 Kerboua, K., O. Hamdaoui. Ultrasonics sonochemistry. 40, 194 (2018).

9 Merouani, S., H. Ferkous, O. Hamdaoui, Y. Rezgui, M. Guemini. Ultrasonics sonochemistry. 22, 51 (2015).

10 Grieser, F., P.-K. Choi, N. Enomoto, H. Harada, K. Okitsu, K. Yasui, eds. Sonochemistry and the Acoustic Bubble. (2015).

11 Brennen, C.E., Cavitation and Bubble Dynamics (2013)

12 Leighton, T.G., The Acoustic Bubble (1994)

13 Yasui, K., Acoustic Cavitation and Bubble Dynamics (2018) 